\title{
A CADAVER STUDY OF THE FUNCTION OF THE OBLIQUE PART OF VASTUS MEDIALIS
}

\author{
JAMES C. H. GOH. PETER Y. C. LEE, KAMAL BOSE \\ From the National University Hospital, Singapore
}

Six normal cadaver lower limbs were mounted on a specially designed loading apparatus. Wires were used to simulate the five muscle bellies of the quadriceps, the ratio of their tensions having been determined from that of the anatomical cross-sectional areas of the muscles. A three-camera system was used to track the patella during knee movements from flexion to extension. The patellofemoral contact area was determined by pressure-sensitive film.

The limb was loaded with and without tension on the wire which simulated the oblique part of the vastus medialis (VMO). Absence of VMO tension caused the patella to displace laterally $(4.2 \mathrm{~mm})$ and increased the load on the lateral patellar facet throughout the range of knee motion. When the tension on the wire simulating vastus lateralis was reduced by $40 \%$ to simulate the effect of a lateral release procedure, the abnormal kinematics caused by the absent VMO returned to normal.

$J$ Bone Joint Surg /Br/ 1995;77-B:225-31.

Received + May 1994: Accepted 22 July 1994

Many patellar disorders are related to abnormalities of patellar tracking (Hughston 1968) but some patients have patellofemoral pain with no obvious mechanical abnormality (Insall 1982). Such symptoms usually arise insidiously and without previous trauma. Ficat and Hungerford (1977) ascribed them to the 'excessive lateral pressure syndrome'. Larson et al (1978) described patients with 'patellar compression syndrome'. In such cases some form of abnormal patellar tracking pattern is thought to exist although it may be rather subtle.

The tracking of the patella is influenced by the shape of the femoral condyles and the patellar articular surface, the

J. C. H. Goh, PhD, CEng. MIMechE, MASME, Senior Research Fellow P. Y. C. Lee. FRCS, MCh Orth. Senior Lecturer and Consultant

K. Bose, MCh Orth, MS, FRCS Ed. AM, Professor and Head of Department

Department of Orthopaedic Surgery. National University Hospital. 5 Lower Kent Ridge Road. Singapore 0511.

Correspondence should be sent to Dr J. C. H. Goh

(1)1995 British Editorial Society of Bone and Joint Surgery (0301-620X/95/2935 \$2.00
Q-angle and the dynamic balance of the medial and lateral components of the quadriceps muscle during contraction (van Kampen 1987). The resultant vector of the forces acting on the patella is normally directed laterally because of the tibiofemoral angle (Huberti and Hayes 1984) and the medialising forces should therefore be important in maintaining the normal alignment of the patella.

Anatomical studies have shown that the inferior portion of the vastus medialis has muscle fibres orientated obliquely, the vastus medialis oblique (VMO) of Lieb and Perry (1968). Bose. Kanagasuntheram and Osman (1980) found at operation that patients with recurrent dislocations of the patella had a deficient VMO and Ahmed. Burke and Yu (1983) showed in cadaver knees that removal of VMO tension shifted the pressure zone from the centre to the lateral facet of the patella.

Our aim was to evaluate the role of the VMO and the effect of lateral release on patellar tracking and the patellofemoral contact pattern.

\section{MATERIALS AND METHODS}

Six fresh left cadaver limbs were used. Each was dissected to expose the five components of the quadriceps and each muscle belly was individually frozen and sectioned at its midpoint perpendicular to the longitudinal axis. The crosssection was traced out on a piece of paper and its area was measured using a digitising tablet (Mypad-A3. Logitec Model K-510 mk2 Digitizer, Kanto Denshi Corporation. Japan). The limb was amputated through the distal third of the femur and all the soft tissues were removed from the distal femur leaving only the collateral and cruciate ligaments intact. The retinacular attachments were dissected off the patella, leaving the patellar tendon intact. All the limbs used for the experiment had normal knees without articular damage.

A steel rod was fixed in the shaft of the femur with bone cement to allow the leg to be mounted on a bench and to hang vertically as shown in Figure 1. No restraining force was applied so that the normal 'screw-home' motion of the tibia on the femur could take place during knee extension.

Five steel wires $(1.2 \mathrm{~mm}$ in diameter) were attached to the patella to simulate each component of the quadriceps. These were passed through pulleys mounted on the bench to reproduce the physiological axis of each muscle (Ahmed et al 1983). Weights were used to provide the required 


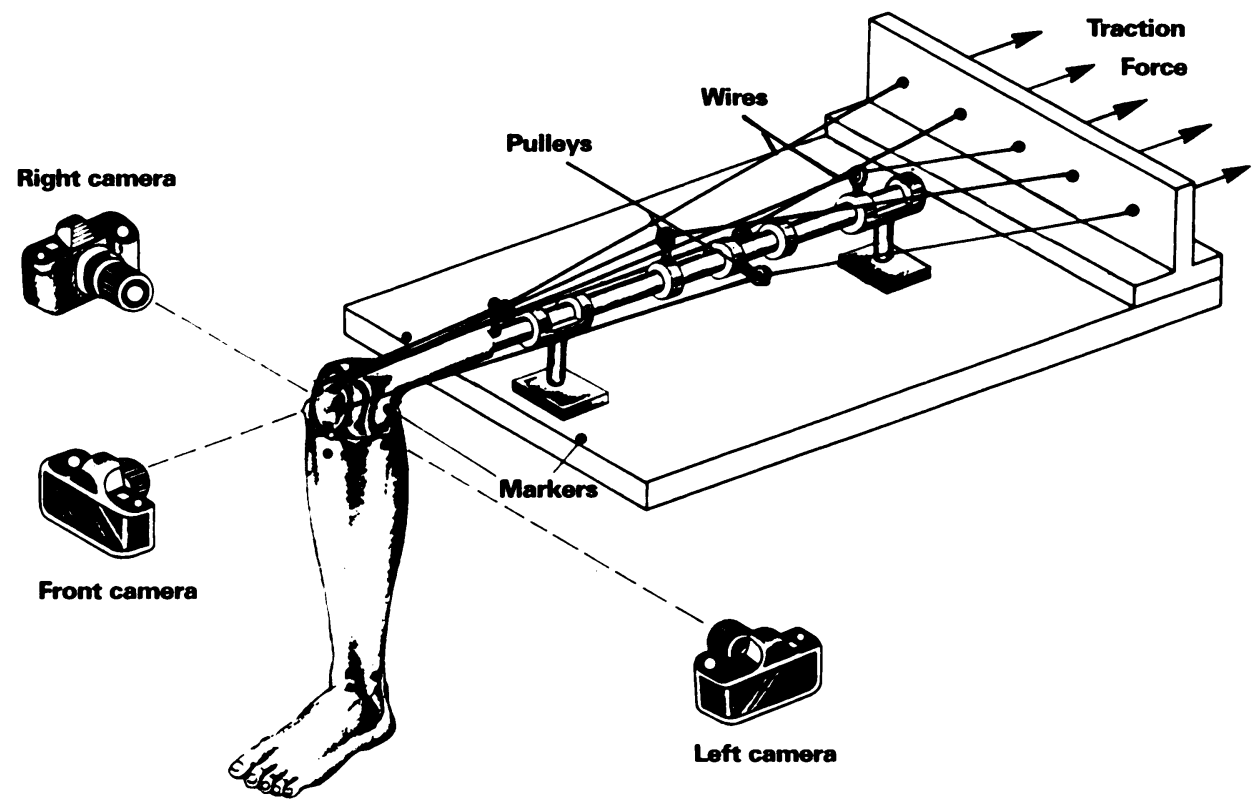

Fig. 1

Diagram of the experimental procedure.

Table I. Mid-muscle cross-sectional areas of the individual bellies of the quadriceps (mean $\pm 1 \mathrm{sD}$ ) and the ratio of each to the rectus femoris. The data are compared with those of Ahmed et al (1983)

\begin{tabular}{|c|c|c|c|}
\hline \multirow{2}{*}{$\begin{array}{l}\text { Muscle } \\
\text { belly }\end{array}$} & \multirow[b]{2}{*}{ Area $\left(\mathrm{cm}^{2}\right)$} & \multicolumn{2}{|l|}{ Ratio } \\
\hline & & Goh et al (1995) & Ahmed et al (1983) \\
\hline Rectus femoris & $13.5 \pm 2.3$ & 1.00 & 1.00 \\
\hline Vastus intermedialis & $15.0 \pm 3.5$ & 1.11 & 1.00 \\
\hline Vastus medialis longus & $12.8 \pm 2.8$ & 0.95 & 1.00 \\
\hline Vastus medialis oblique & $22.6 \pm 3.7$ & 1.67 & 1.50 \\
\hline Vastus lateralis & $38.3 \pm 4.8$ & 2.84 & 2.50 \\
\hline
\end{tabular}

tensions, the ratio of the tension in each muscle belly having been calculated from its cross-sectional area and compared with that of the rectus femoris (Table I).

Luminous markers $(1.5 \mathrm{~mm}$ in diameter) were placed on the medial and lateral femoral condyles, the tibial tubercle, the fibular head, the lateral malleolus, the superior and inferior poles and the medial and lateral borders of the patella. Two reference markers were attached to the front edge of the bench and one on top of the steel rod (Fig. 1).

Three motorised cameras (Nikon F501) were used, one situated anteriorly and the other two on either side of the specimen in an orthogonal arrangement (Fig. 1). A frame of reference was established with its origin at the midpoint of the front edge of the bench. The cameras' shutters were linked to a single trigger to capture the position of the knee instantaneously in the three views. The system was calibrated using a wire-frame cube $(20 \mathrm{~cm} \times 20 \mathrm{~cm} \times 20 \mathrm{~cm})$ of 16 markers. The $35 \mathrm{~mm}$ photographic slides were projected on to a Mypad-A3 digitising tablet. The markers' positions were digitised sequentially and the data were stored in an IBM-compatible 386 personal computer. A program was written to correct for lens distortion and parallax errors in the data.

The three markers on the leg were used to define the motion of the rigid body segment and the axis vector of the leg was determined using matrix methods (Pipes 1963). The femoral axis vector was defined by the markers on the femoral condyles and on the steel rod. The knee angles were calculated from the cross-product of these two axis vectors. The patellar axes and motion were determined from the four patellar markers and the markers on the femoral condyles (Fig. 2).

We studied patellar flexion (patellar angulation in the sagittal plane), patellar tilt (patellar angulation about its caudocranial axis), patellar rotation (rotation in the coronal plane) and patellar shift (mediolateral translation of the patella in the coronal plane). 


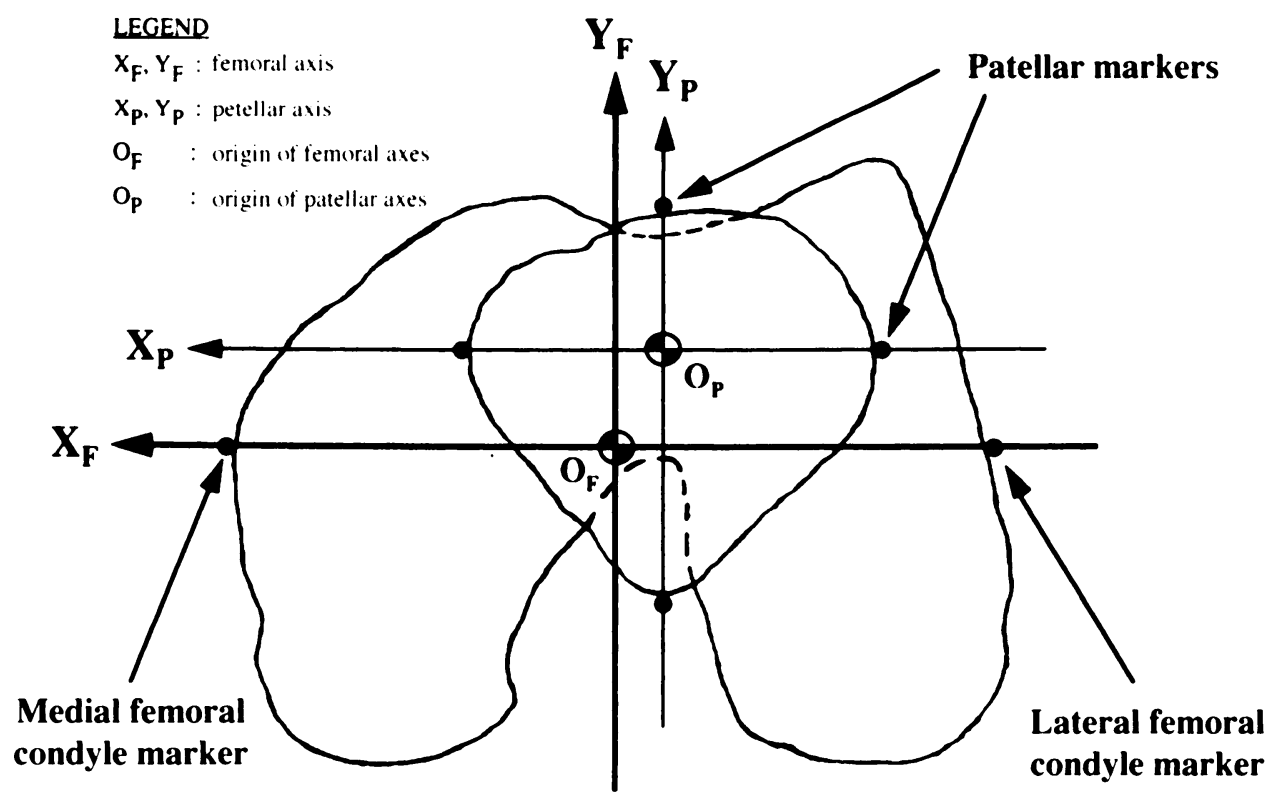

Fig. 2

The femoral and patellar axes as defined by the four patellar markers and the markers on the femoral condyles.

Patellar tracking. The joint surfaces were sprayed constantly with Ringer's solution. The leg was first photographed in the neutral position (i.e., hanging at $90^{\circ}$ flexion). Weights were then added to each wire in the ratio given in Table I and the leg was allowed to oscillate until it came to rest. The new position of the leg was then photographed. The procedure was repeated with each increment of weights until nearly full extension of the knee was achieved. Data were recorded between knee flexion angles of $90^{\circ}$ and $20^{\circ}$. Measurement at less than $20^{\circ}$ of knee flexion was not possible because the markers were obscured and could not be photographed by the cameras.

Patellofemoral contact. Pressure-sensitive films (Fuji Prescale Film. Pressure Grade Low, Fuji Photo Film Co Ltd, Japan) were used to study the contact areas at $30^{\circ}$ and $60^{\circ}$ of flexion. The film was cut to the size of the patella and sealed in a plastic envelope. The wire tensions needed to achieve the desired knee flexion angles were determined from the previous experiments. As in the patellar tracking study, the leg was allowed to come to rest after the application of the weights. It was then held in that position, the tension in the wires was released and the pressure-sensitive film was inserted between the patella and the femoral condyles. The patella was then repositioned and the wires again put under tension. Care was taken to ensure that no sliding movements of the patella occurred at this stage, and if they did the sequence was repeated with a new film. The outline of the patella and the positions of the four patellar markers were traced on the film. the contact areas were digitised and a simple program was used to calculate the areas.

Experimental groupings. Three types of experiment were carried out in sequence on each specimen, as follows:
Group 1 (normal). The first sequence was to simulate normal knee function. The wire tensions were applied according to the ratio given in Table $\mathrm{I}$.

Group 2 (without VMO). The second sequence was to simulate knees with a deficient VMO. The wire simulating the VMO was not loaded but the tensions in the other wires were maintained in the ratio described.

Group 3 (without VMO and with reduced VL tension). The third sequence was to simulate knees with a deficient VMO after a lateral release procedure had been performed. The wire simulating the VMO was not loaded and the tension on the wire simulating the VL was reduced by $40 \%$.

\section{RESULTS}

The measuring system was found to be accurate to $\pm 1.5 \mathrm{~mm}$ in the calibration volume and the calculation of the angles was accurate to $\pm 2^{\circ}$.

The mean peak quadriceps tension (the sum of the tensions in the five wires) was $198.5 \pm 12.6 \mathrm{~N}$ at full extension, $172.5 \pm 9.6 \mathrm{~N}$ at $30^{\circ}$ flexion and $130.0 \pm 10.4 \mathrm{~N}$ at $60^{\circ}$ flexion.

Figures 3 to 6 summarise the results of the three experiments performed in sequence on one specimen.

\section{Patellar tracking}

Group $I$ (normal). At $90^{\circ}$ of knee flexion, the patellar flexion angle was between $65^{\circ}$ and $75^{\circ}$. As the knee extended the patellar flexion angle decreased. On average. patellar flexion was found to lag behind knee flexion by about $20^{\circ}$ to $30^{\circ}$ throughout the range of motion analysed (Fig. 3).

At $90^{\circ}$ of knee flexion. the patella was in a position of lateral rotation (Fig. 4). As the knee extended, the patella 


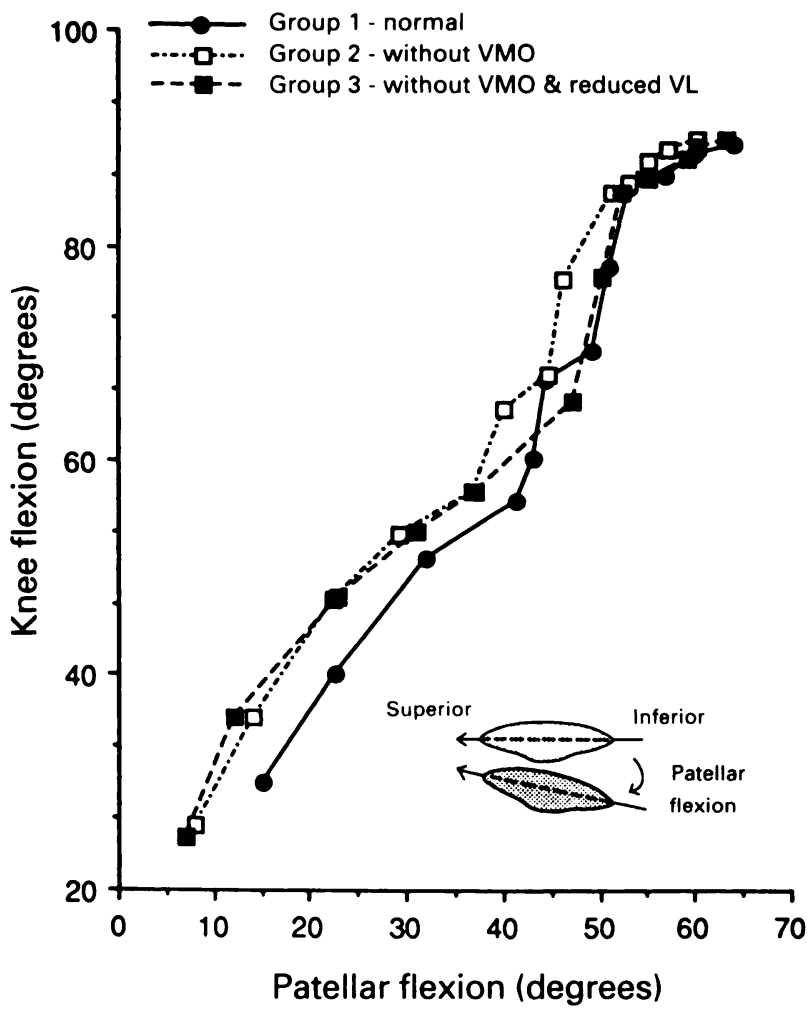

Fig. 3

Relationship of the patellar flexion angle to knee flexion angle.

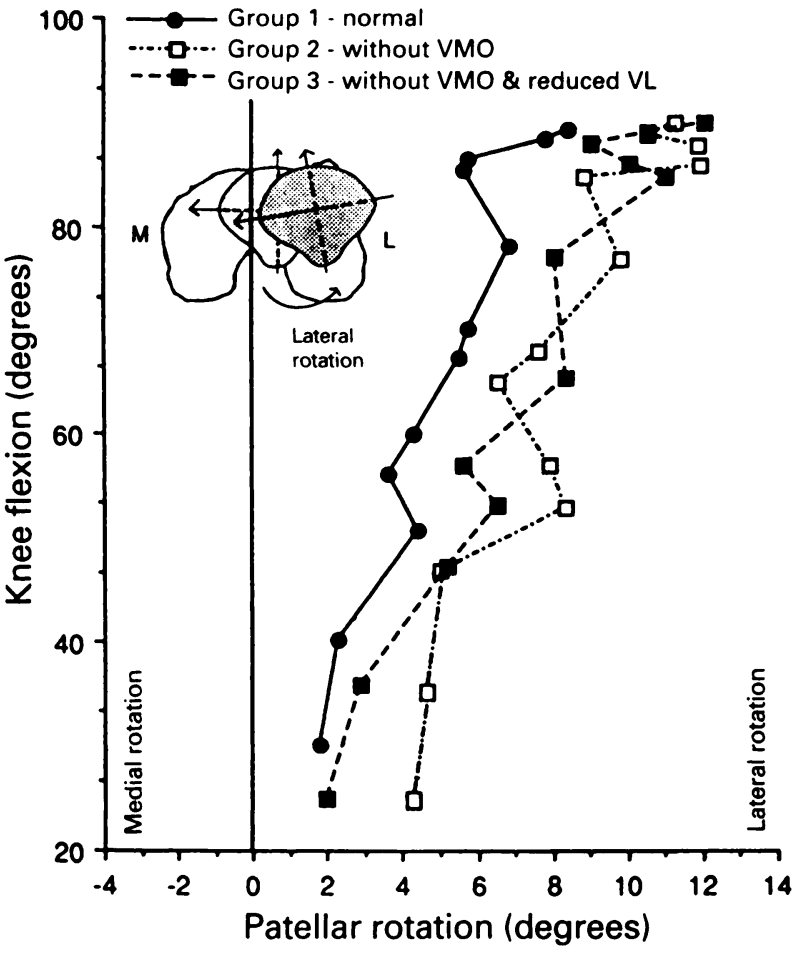

Fig. 4

Relationship of the patellar rotation angle to knee flexion angle.

rotated medially, decreasing the lateral patellar rotation angle by about $6^{\circ}$.

Patellar tilt was found to be inconsistent between specimens. The general pattern, however, was that as the knee extended from $90^{\circ}$ of flexion, the patella tilted laterally from a neutral or medially-rotated position (Fig. 5). With further knee extension, it tilted medially again, and towards the end of knee extension it was either in a neutral or a medially-rotated position. The mean overall tilt angle was $5.4 \pm 3^{\circ}$ laterally.

At $90^{\circ}$ of knee flexion, the patella was lateral to the midline of the femoral condyles (Fig. 6). As the knee extended it shifted medially but with further knee extension it changed course and shifted slightly laterally. The mean overall shift was $5.5 \pm 2.1 \mathrm{~mm}$ medially.

Group 2 (without VMO). No difference was found in patellar flexion when VMO tension was removed (Fig. 3). Patellar rotation was similar to that of group 1, except that in nearly full extension, there was less medial rotation (Fig. 4). Mediolateral tilt was also similar to that of group 1, except that the degree of lateral tilt had increased, although not significantly (Fig. 5).

At $90^{\circ}$ flexion, the patella was displaced more laterally than in group $1(4.2 \pm 1.8 \mathrm{~mm})$ (Fig. 6). The shift pattern was similar to that of group 1 but medial shift during extension was less. At nearly full extension the mean overall shift was $4.1 \pm 1.5 \mathrm{~mm}$ medially.

Group 3 (without VMO and with reduced VL tension). The patterns of patellar flexion, rotation, tilt and shift were similar to those of group 1 (Figs 3 to 6).

Patellofemoral contact areas. Figure 7 shows the typical patellofemoral contact patterns of one specimen in all three experiments.

Group 1 (normal). The contact occupied $20 \%$ and $27 \%$ of the patellar articular surface area at $30^{\circ}$ and $60^{\circ}$ of flexion, respectively (Table II). The region of the superior pole of the patella was in contact at $60^{\circ}$ flexion and as extension progressed the contact area migrated inferiorly so that at $30^{\circ}$ it was at the mid-region of the patella. At $30^{\circ}$ flexion, $65 \%$ of the area of contact was on the lateral facet and at $60^{\circ}, 35 \%$ was on the lateral facet.

Group 2 (without VMO). The contact area on the medial facet was significantly reduced to about $8 \%$ while that on the lateral facet was greatly increased, up to about $92 \%$. The total contact area was less than that of the normal.

Group 3 (without VMO and with reduced VL tension). The contact area pattern was similar to that of group 1, except that the distribution of contact between the medial and lateral facets was more even. The total area of contact was near to normal.

\section{DISCUSSION}

The terms used in the past to describe patellar kinematics have been confusing and inconsistent making comparison of data from different studies difficult. The use of Cartesian co-ordinates was proposed by van Kampen (1987) and has been adapted in this study. Another difficulty is that different studies have employed different experimental designs. In some, the forces applied were isometric, in some iso- 


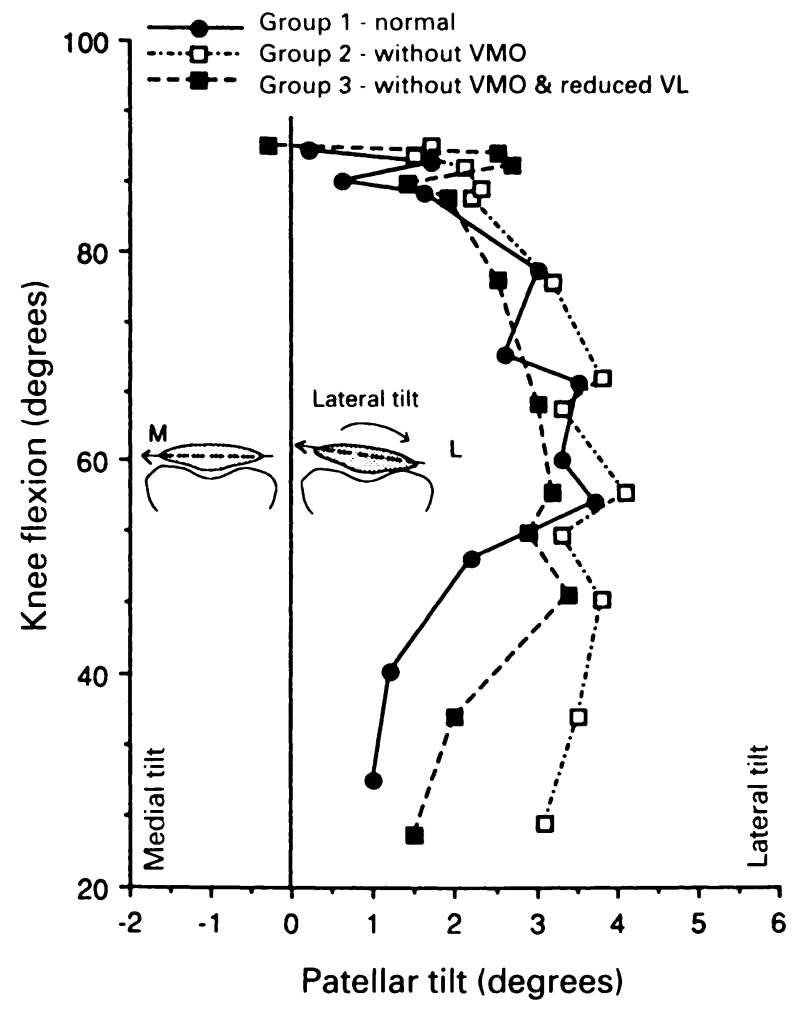

Fig. 5

Relationship of the patellar tilt angle to knee flexion angle.

tonic and in some the limb was restrained and in others not. Furthermore, there were differences in the quadriceps tension used and in the methods of simulating the tension (single or multiple components). Some studies tested the knee in flexion, others in extension.

The use of the cross-sectional area of an individual muscle belly to estimate its tension has not been fully validated, although Ikai and Fukunaga (1968) reported that maximum tension and cross-sectional area are directly proportional. According to Winter (1979), if muscles of the same group share a common load they probably do so proportionally to their relative cross-sectional areas. Ahmed et al (1983), however, cautioned that the tensions in the individual components of the quadriceps may not accord with their cross-sectional areas throughout the range of movements. Furthermore, the 'anatomical' cross-sections taken from the cadaver do not take account of the differences in angulation within the muscle, i.e., the physiological cross-section. With insufficient information to validate the method and lack of a more suitable method available, we used cross-sectional areas as a rough approximation.

All cadaver studies have the limitation that it is difficult to reproduce the exact physiological conditions. In our study, for instance, the soft tissues surrounding the knee were removed, including the patellar retinaculum, on the assumption that they had a uniform effect on the patella, and that removing them would not alter the tension dis-

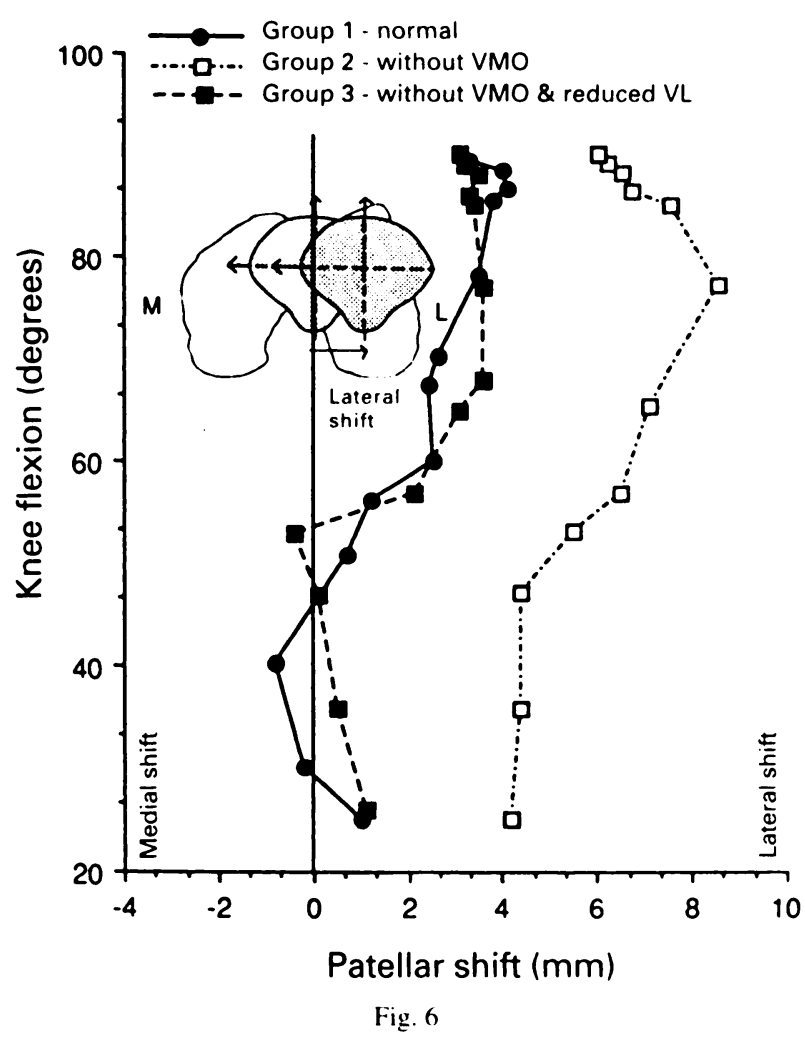

Relationship of the patellar shift to knec flexion angle. $60^{\circ}$ Knee flexion $30^{\circ}$ Knee flexion
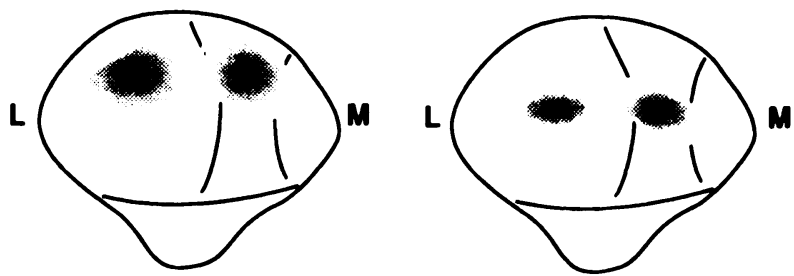

a) Group 1 - normal
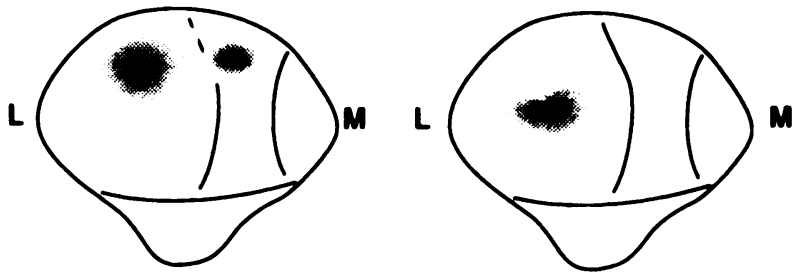

b) Group 2 - without VMO

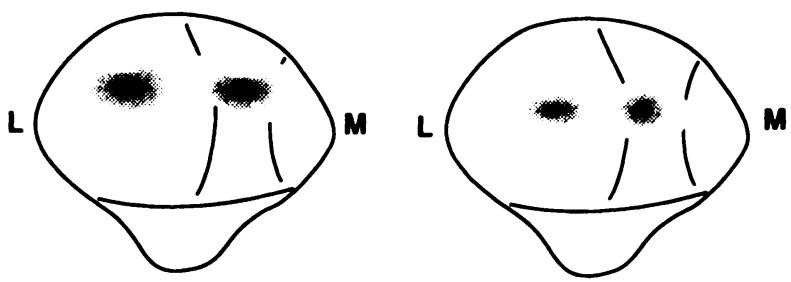

c) Group 3 - without VMO and reduced VL

Fig. 7

Pattern of patellofemoral contact areas at 30 and 6$)^{\circ}$ knee flexion 
Table II. Patellar contact area (mean $\pm 1 \mathrm{SD}$ ) at $30^{\circ}$ and $60^{\circ}$ knee flexion

\begin{tabular}{|c|c|c|c|c|c|c|}
\hline & \multicolumn{3}{|l|}{$60^{\circ}$ flexion } & \multicolumn{3}{|l|}{$30^{\circ}$ flexion } \\
\hline & $\begin{array}{l}\text { Contact } \\
\operatorname{area}\left(\mathrm{cm}^{2}\right)\end{array}$ & $\begin{array}{l}\text { Total } \\
\text { contact }(\%)\end{array}$ & $\begin{array}{l}\text { Lateral } \\
\text { contact }(\%)\end{array}$ & $\begin{array}{l}\text { Contact } \\
\operatorname{area}\left(\mathrm{cm}^{2}\right)\end{array}$ & $\begin{array}{l}\text { Total } \\
\text { contact }(\%)\end{array}$ & $\begin{array}{l}\text { Lateral } \\
\text { contact }(\%)\end{array}$ \\
\hline Group 1* & $3.1 \pm 0.4$ & 27.3 & 65.9 & $2.4 \pm 0.4$ & 20.3 & 64.5 \\
\hline Group 2* & $2.6 \pm 0.6$ & 23.4 & 90.9 & $1.9 \pm 0.4$ & 17.9 & 93.4 \\
\hline Group 3* & $2.8 \pm 0.4$ & 25.8 & 56.7 & $2.3 \pm 0.5$ & 19.5 & 51.8 \\
\hline
\end{tabular}

tribution. The use of wires to simulate muscle force vectors is a reasonable model but care must be taken to ensure that their attachment to the patella is accurate and that their direction of pull is appropriate. The simulation of VMO weakness by not pulling on the VMO wire represented extreme pathology, and the $40 \%$ reduction of the VL tension to simulate the effects of a lateral release may be criticised since lateral release surgery is designed primarily to realign the lateral force vector rather than to weaken the VL. It is therefore necessary to view results from such studies with care, taking into account the assumptions made and the inherent limitations of the experimental method.

In our study, the normal patellar kinematics during knee extension were characterised by a decreasing patellar flexion angle, decreasing lateral rotation, lateral tilt, and medial shift of an initially laterally placed patella. The patellar flexion angles lagged behind the knee flexion angles. These findings are similar to those of van Ejiden (1985) and van Kampen (1987), although they studied flexion rather than extension. Removal of the VMO caused no appreciable change, probably because the VMO contributes very little to the extension force that causes patellar flexion (Lieb and Perry 1968).

From its laterally rotated position at $90^{\circ}$ flexion the patella moved during extension into medial rotation as was observed by Reider, Marshall and Ring (1981). This patellar rotation occurs as the patella rides up the condylar groove, which itself slopes laterally from below to above. Removal of the VMO did not therefore alter the patellar rotation pattern until nearly full extension when medial rotation was slightly decreased.

Patellar tilt during knee flexion was described by van Kampen (1987) as wavering, i.e., varying from medial tilt to lateral tilt and then back again. Reider et al (1981) reported an average medial tilt of $12^{\circ}$, from $90^{\circ}$ of flexion to full extension, more than was observed in our study. With the VMO removed, the medial tilt was reduced.

Reider et al (1981) also reported a medial shift of the patella averaging $14 \mathrm{~mm}$ ( 7 to 21 ) during knee extension and van Kampen (1987) reported a medial shift which ranged from $4.9 \mathrm{~mm}$ to $16.2 \mathrm{~mm}$. The shift observed by us was much less, ranging from $3.3 \mathrm{~mm}$ to $8.1 \mathrm{~mm}$. With the VMO removed, the average medial shift was reduced by $4.2 \mathrm{~mm}$ compared with the normal.

Removal of the medial force applied by the VMO results in a more laterally directed tension in the quadriceps and a greater load on the lateral facet. This effect was noted at both $30^{\circ}$ and $60^{\circ}$ of flexion. Ahmed et al (1983) found that with the VMO removed, the pressure zone shifted entirely to the lateral facet of the patella.

Lieb and Perry (1968) and Bose et al (1980) thought that the VMO was of significance mainly in the last phases of extension and our experiments support this belief. In the absence of the VMO significant deviation from the normal kinematics occurred only at nearly full extension. The patellofemoral contact studies, however, indicate that the VMO plays an important role in controlling the contact area and pressure distribution throughout the range of knee motion. There was evidence of excessive contact pressure when the VMO was absent, even when the patellar kinematics at nearly full extension appeared relatively normal.

Lateral release is meant to alter the pressure distribution in the patellofemoral joint, by medialising the resultant quadriceps force but previous studies have not shown that the procedure has a significant effect on contact patterns and pressures. Van Kampen (1987) observed that the patellar tracking pattern did not change significantly after lateral retinaculum release; it only influenced the patellar position at nearly full extension. In our study a large reduction $(40 \%)$ of the lateral vector was required to counteract the lateral displacement of the patella caused by an absent VMO. In clinical practice lateral retinaculum release may not achieve a similar reduction in the lateral vector and were such a $40 \%$ reduction to be achieved it would greatly weaken the quadriceps as a whole and possibly result itself in a functional deficit. A more logical approach therefore may be to determine the minimum reduction of the lateral vector required and/or to improve the medial force vector by muscle-strengthening programmes.

Conclusion. During flexion/extension of the knee the patella rotates about three orthogonal axes and translates mediolaterally and inferosuperiorly. The kinematic pattern is influenced by the contours of the femoral condyles and the patellar articular surface, the tibiofemoral angle and the resultant vector of the quadriceps muscle.

The VMO is a distinct muscle within the quadriceps group, deficiency of whose function results in abnormal kinematics of the patella with alteration of the patellofemoral contact area and increase in load on the lateral patellar facet throughout the range of knee motion. 
A simulated lateral release procedure in which the lateral force was reduced by $40 \%$, restored the normal kinematics.

The authors wish to thank Mr Ang Eng Joo, Ms Grace Lee and Mr Hazlan Sanusi for their dedicated assistance in carrying out this project. Funding was provided by the National University of Singapore Research Grant (RP 830065).

No benefits in any form have been received or will be received from a commercial party related directly or indirectly to the subject of this article.

\section{REFERENCES}

Ahmed AM, Burke DL, Yu A. In-vitro measurement of static pressure distribution in synovial joints-Part Il: retropatellar surfaces. $J$ Biomech Eng 1983;105:226-36.

Bose K, Kanagasuntheram R, Osman MBH. Vastus medialis oblique: an anatomic and physiologic study. Orthopedics 1980:3:880-3.

van Ejiden TGML. The mechanical behaviour of the patellofemoral joint. Dissertation. University of Amsterdam, 1985.

Ficat RP, Hungerford DS. Disorders of the patello-femoral joint. Paris: Masson, 1977.
Huberti HH, Hayes WC. Patellofemoral contact pressures: the influence of Q-angle and tendofemoral contact. J Bone Joint Surg [Am] 1984;66-A:715-24.

Hughston JC. Subluxation of the patella. J Bone Joint Surg (Am] 1968;50-A:1003-26.

Ikai M, Fukunaga T. Calculation of muscle strength per unit crosssectional area of human muscle by means of ultrasonic measurement. Int $Z$ agnew Physiol sinschi Arbeitphysiol 1968;26:26-32.

Insall J. Patellar pain. J Bone Joint Surg [Am] 1982;64-A:147-52.

van Kampen $A$. The three-dimensional tracking pattern of the patella. Dissertation, University of Nijmegen, 1987.

Larson RL, Cabaud HE, Slocum DB, et al. The patellar compression syndrome: surgical treatment by lateral retinacular release. Clin Orthop 1978:134:158-67.

Lieb FJ, Perry J. Quadriceps function: an anatomical and mechanical study using amputated limbs. J Bone Joint Surg [Am/ 1968; 50-A:1535-48.

Pipes LA. Matrix methods for engineering. Englewood Cliffs, UK: Prentice-Hall, 1963.

Reider B, Marshall JL, Ring B. Patellar tracking. Clin Orthop 1981:157:143-8.

Winter DA. Biomechanics of human movement. Canada: A Wiley-Interscience Publication, 1979. 